TITLE:

\title{
Kinetics of shrinking of polymer gels induced by ultracentrifugal fields
}

\section{$\operatorname{AUTHOR}(\mathrm{S})$ :}

Urayama, K; Okada, S; Nosaka, S; Watanabe, H; Takigawa, T

\section{CITATION:}

Urayama, K ... [et al]. Kinetics of shrinking of polymer gels induced by ultracentrifugal fields. JOURNAL OF CHEMICAL PHYSICS 2005, 122 (2): 024906.

\section{ISSUE DATE:}

2005-01-08

URL:

http://hdl.handle.net/2433/39761

\section{RIGHT:}

Copyright 2005 American Institute of Physics. This article may be downloaded for personal use only. Any other use requires prior permission of the author and the American Institute of Physics. 


\title{
Kinetics of shrinking of polymer gels induced by ultracentrifugal fields
}

\author{
Kenji Urayama \\ Department of Material Chemistry, Kyoto University, Kyoto 615-8501, Japan \\ Shinichi Okada \\ Institute for Chemical Research, Kyoto University, Uji, Kyoto 611-0011, Japan \\ Shoji Nosaka \\ Department of Material Chemistry, Kyoto University, Kyoto 615-8501, Japan \\ Hiroshi Watanabe \\ Institute for Chemical Research, Kyoto University, Uji, Kyoto 611-0011, Japan \\ Toshikazu Takigawa ${ }^{\text {a) }}$ \\ Department of Material Chemistry, Kyoto University, Kyoto 615-8501, Japan
}

(Received 3 August 2004; accepted 28 October 2004; published online 21 December 2004)

\begin{abstract}
The kinetics of the shrinking of polymer gels induced by ultracentrifugal fields is investigated. A theory is proposed to describe the diffusion process of polymer networks under centrifugal fields. The initial shrinking rate is proportional to the ratio of the centrifugal force to the frictional force of networks. The shrinking attains the stationary state as a result of the balance between the centrifugal force and the swelling force of networks. The characteristic time for shrinking is of the order of $a^{2} / D$ where $a$ and $D$ are the stationary displacement and diffusion constant, respectively. We also present the experimental data for the shrinking of the poly(acrylamide) (PAAm) gels under ultracentrifugal fields. The shrinkage increases linearly with time in the initial stage whereas it reaches the steady state in the long time limit as expected by the theory. Each of longitudinal elastic modulus and friction coefficient of the PAAm gels is evaluated from the data on the basis of the theory. (C) 2005 American Institute of Physics. [DOI: 10.1063/1.1835912]
\end{abstract}

\section{INTRODUCTION}

The kinetics of swelling and shrinking of polymer networks in solvents (gels) has been analyzed on the basis of diffusion models of networks. ${ }^{1-6}$ As is pointed out in Ref. 7, the diffusion models without considering a stress-diffusion coupling effect cannot provide the exact solution for the time dependence of swelling and shrinking. However, the diffusion models still have the advantage that the mathematical treatments for the kinetics of swelling and shrinking are facilitated whereas the models yield a solution with practically sufficient accuracy. On the basis of the diffusion models, the characteristic time for swelling and shrinking is of the order of $d^{2} / D$, where $d$ and $D$ are the final dimension of the gel and the diffusion constant of the network, respectively. The constant $D$ is governed by the elastic and frictional characteristics of networks as $D=L / f$, where $L$ and $f$ are the longitudinal elastic modulus and friction coefficient between network and solvent, respectively. The elastic moduli such as Young's modulus and shear modulus can be easily measured by conventional mechanical testing. By contrast, there exist only a few studies ${ }^{1,89}$ on the measurements of $f$ due to the limited experimental methods.

The dynamics of swelling and shrinking of gels has been investigated under no external field ${ }^{1-4}$ as well as under static strains ${ }^{7,10-15}$ or dynamic loads. ${ }^{16}$ The present study focuses

\footnotetext{
a) Author to whom all correspondence should be addressed. Electronic mail: takigawa@rheogate.polym.kyoto-u.ac.jp
}

on the shrinking behavior of gels under constant ultracentrifugal fields which has not been reported before. A theory based on the diffusion model is proposed to describe the kinetics of the shrinking of gels driven by centrifugal fields. The theory shows that the characteristic time for shrinking is proportional to $a^{2} / D$ where $a$ is the displacement in the stationary state, and that the initial shrinking rate is determined by the ratio of the centrifugal force to the frictional force acting on networks whereas the stationary state of shrinking is achieved in the long time limit by the balance between the centrifugal force and the swelling force of networks. We also present the experimental data for the shrinking behavior of polyacrylamide gels in water under ultracentrifugal fields. The whole shrinking process of the gels observed is compared by the theoretical prediction. Each of longitudinal modulus and friction coefficient of the network is evaluated from the stationary shrinkage and initial shrinking rate, respectively.

\section{THEORY}

\section{A. Basic equations}

According to the diffusion model introduced by Tanaka et al., ${ }^{1}$ the equation of motion for a unit volume element in the network with density $\rho_{N}$ under an external field is generally written in the Cartesian coordinates $\left(O-x_{1} x_{2} x_{3}\right)$ as

$$
\phi \rho_{N} \frac{\partial^{2} \mathbf{u}}{\partial t^{2}}=\nabla \cdot \sigma-f \frac{\partial \mathbf{u}}{\partial t}
$$




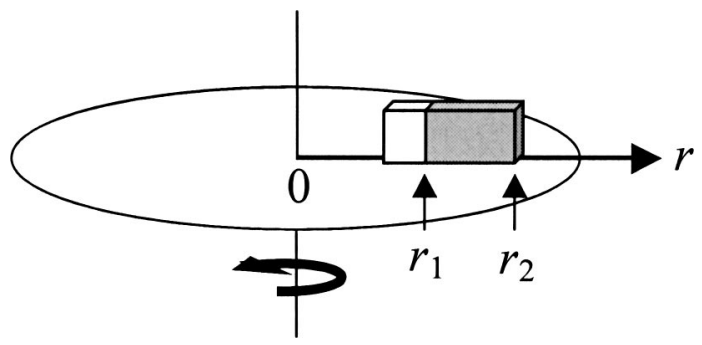

FIG. 1. Schematic for a gel under centrifugal fields. The gray part indicates a gel confined in a cell. Each of $r_{1}$ and $r_{2}$ corresponds to the position of the moving boundary (gel surface) and immobile bottom wall of the cell.

where $\mathbf{u}$ is the displacement vector representing the displacement of a point from the initial position at $t=0$, and $\phi$ is the volume fraction of the network. When the motion is described in terms of the rotating system of coordinates, the equation generally becomes complicated. However, if the rotation occurs around a fixed axis (for example, the $x_{3}$-axis being the fixed one) with a constant angular velocity, the equation has a rather simple form:

$$
\phi \rho_{N} \frac{\partial^{2} \mathbf{u}}{\partial t^{2}}=\nabla \cdot \sigma-f \frac{\partial \mathbf{u}}{\partial t}+\mathbf{F} .
$$

Here, $\mathbf{F}$ denotes the apparent force: the sum of the centrifugal and Coriolis forces. The term on the left-hand side of the equation can be neglected when the motion of the volume element is sufficiently slow in the time scale concerned: The time scale treated here is still longer than the really short time region where the inertia term becomes significant. On the basis of the linear elasticity theory, in the Cartesian coordinates, $\boldsymbol{\sigma}$ in the above equations is related to $\mathbf{u}$ as ${ }^{17}$

$$
\sigma_{i j}=\left(K_{\mathrm{os}}-\frac{2}{3} G\right) \nabla \cdot \mathbf{u} \delta_{i j}+G\left(\frac{\partial u_{i}}{\partial x_{j}}+\frac{\partial u_{j}}{\partial x_{i}}\right),
$$

where $K_{\mathrm{os}}$ and $G$ are the osmotic bulk modulus and shear modulus of the network alone, respectively.

We now consider a simple case illustrated in Fig. 1. A polymer gel (polymer network swollen in solvent) confined in a rectangular cell is placed on a rotating axis $\left(x_{1}\right.$-axis) which is hereafter renamed as the $r$-axis. The cell rotates at a constant angular frequency $\omega$. Namely, the system considered here is that a gel (in an actual case, the sample cell) moves on an orbit. When the interface between gel and side wall of the cell is a slip boundary, the network is deformable only in the $r$-direction while no deformation occurs in the other directions. As a result this shrinking process can be treated as the one-dimensional (1D) motion of network, which gives the following equation from Eqs. (1b) and (2),

$$
\frac{\partial u(r, t)}{\partial t}=D \frac{\partial^{2} u(r, t)}{\partial r^{2}}+\frac{\phi \Delta \rho \omega^{2}}{f} r .
$$

Here, the inertia term is eliminated. The constant $D$ is defined by $D=(K+4 G / 3) / f=L / f$ with $L$ being the longitudinal elastic modulus. In Fig. 1, the center of rotation, the gel surface in the initial state and the bottom of the cell correspond to $r=0, r=r_{1}$, and $r=r_{2}$, respectively. The second term in the right-hand side of Eq. (3) originates from a cen- trifugal field. The centrifugal force acting on the element at distance $r$ from the origin is proportional to $r \omega^{2}$, the volume fraction of network $\phi$, and the difference in density $\Delta \rho$ between polymer network and solvent.

\section{B. Solution}

The initial condition to solve Eq. (3) is given by

$$
u(r, 0)=0 \quad \text { at } t=0 .
$$

The boundary conditions at the surface $\left(r=r_{1}\right)$ and bottom $\left(r=r_{2}\right)$ of the cell may be, respectively, given by

$$
\frac{\partial u\left(r_{1}, t\right)}{\partial r}=0 \quad \text { at } r=r_{1}
$$

and

$$
u\left(r_{2}, t\right)=0 \quad \text { at } r=r_{2} .
$$

Equation (5) originates from that the stress normally acting on the gel surface (moving surface) is zero. ${ }^{2}$ The solution of Eq. (3) satisfying the initial and boundary conditions is

$$
u(r, t)=\sum_{n=1}^{\infty} A_{n} \exp \left(-\frac{(2 n-1)^{2} t}{\tau_{L}}\right)+u(r, \infty)
$$

with

$$
\begin{aligned}
A_{n}(r)= & \frac{16 a^{2} \phi \Delta \rho \omega^{2}}{\pi^{4} L}\left\{\frac{(-1)^{n} \pi r_{2}}{(2 n-1)^{3}}+\frac{2 a}{(2 n-1)^{4}}\right\} \\
& \times \cos \left\{\frac{(2 n-1) \pi\left(r-r_{1}\right)}{2 a}\right\},
\end{aligned}
$$

where $a=r_{2}-r_{1}$. In Eq. (7a), $\tau_{L}$ is the longest characteristic time defined by

$$
\tau_{L}=\frac{4 a^{2}}{\pi^{2} D} .
$$

The term $u(r, \infty)$ in Eq. (7a) is the stationary value of $u$ in the long time limit,

$$
u(r, \infty)=\frac{\phi \Delta \rho \omega^{2}}{6 L}\left(r_{2}^{3}+3 r_{1}^{2} r-3 r_{1}^{2} r_{2}-r^{3}\right) .
$$

In particular, the displacement of the moving surface in the stationary state $u\left(r_{1}, \infty\right)$ is given by

$$
u\left(r_{1}, \infty\right)=\frac{\phi \Delta \rho \omega^{2}}{6 L}\left(2 r_{1}^{3}-3 r_{1}^{2} r_{2}+r_{2}^{3}\right) .
$$

Equations (9) and (10) indicate that the shrinkage in the steady state is determined by the balance between the centrifugal force and the swelling force. Meanwhile, in the initial stage of shrinking at $t / \tau_{L} \ll 1, u\left(r_{1}, t\right)$ is approximated only by a linear term of $t$ in a series expansion of Eq. (7) around $t=0$ as

$$
u\left(r_{1}, t\right)=\frac{\phi \Delta \rho r_{1} \omega^{2}}{f} t \text { for } t / \tau_{L} \ll 1 .
$$

Equation (11) shows that the initial shrinking rate is proportional to the ratio of the centrifugal force to the frictional 


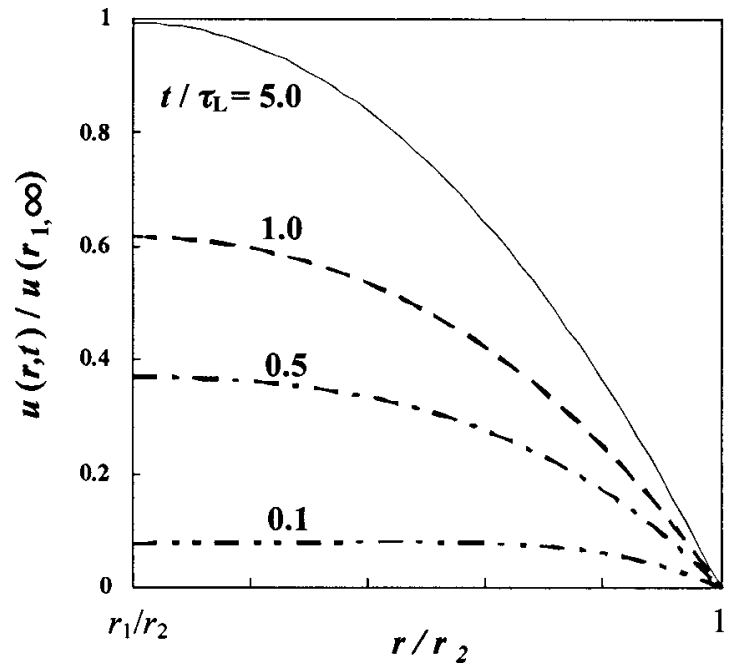

FIG. 2. The displacement distribution of a gel during shrinking. The relative position $u(r, t) / u\left(r_{1}, \infty\right)$ is displayed as a function of $r / r_{2}$ at various reduced time $t / \tau_{L}$. In the calculation, $r_{1}=6 \times 10^{-2} \mathrm{~m}$ and $r_{2}=7 \times 10^{-2} \mathrm{~m}$ are employed.

force. Thus the shrinking behavior in the short or long time limit strongly reflects the frictional or elastic property of gels, respectively.

Figure 2 illustrates $u(r, t) / u\left(r_{1}, \infty\right)$ as a function of $r / r_{2}$ at various reduced times $t / \tau_{L}$, i.e., the displacement distribution of a gel during shrinking. In the calculation, $r_{1}=6$ $\times 10^{-2} \mathrm{~m}$ and $r_{2}=7 \times 10^{-2} \mathrm{~m}$; both of which are close to the experimental condition in present study, were employed. In the initial state $t=0, u(r, 0)=0$. The profile of the stationary displacement is given by a cubic function of $r$ of Eq. (9).

Figure 3 displays the displacement of the moving surface $u\left(r_{1}, t\right)$ at various centrifugal accelerations $\alpha\left(=r_{1} \omega^{2} / g\right.$ where $g$ is the acceleration of gravity) as a function of reduced time $t / \tau_{L}$. The calculation was performed with $r_{1}$ $=6 \times 10^{-2} \mathrm{~m}, \quad r_{2}=7 \times 10^{-2} \mathrm{~m}, \quad L=3 \times 10^{4} \mathrm{~Pa}, \quad f=1$ $\times 10^{14} \mathrm{Ns} / \mathrm{m}^{4}, \phi=2 \times 10^{-2}$, and $\Delta \rho=4.48 \times 10^{2} \mathrm{~kg} / \mathrm{m}^{3}$. As

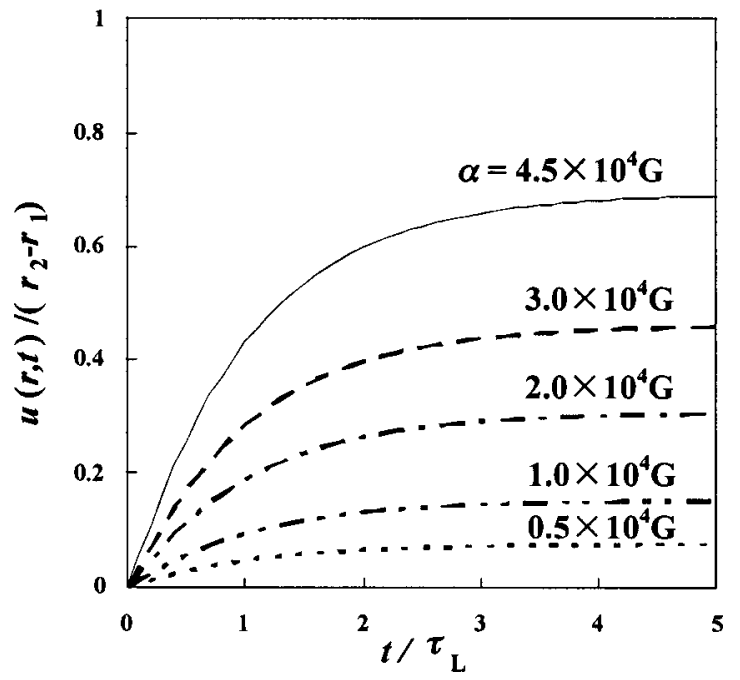

FIG. 3. The shrinking behavior of a gel at various centrifugal accelerations $(\alpha)$. The calculation is performed with $r_{1}=6 \times 10^{-2} \mathrm{~m}, r_{2}=7 \times 10^{-2} \mathrm{~m}, L$ $=3 \times 10^{4} \mathrm{~Pa}, f=1 \times 10^{14} \mathrm{Ns} / \mathrm{m}^{4}, \phi=2 \times 10^{-2}, \Delta \rho=4.48 \times 10^{2} \mathrm{~kg} / \mathrm{m}^{3}$.

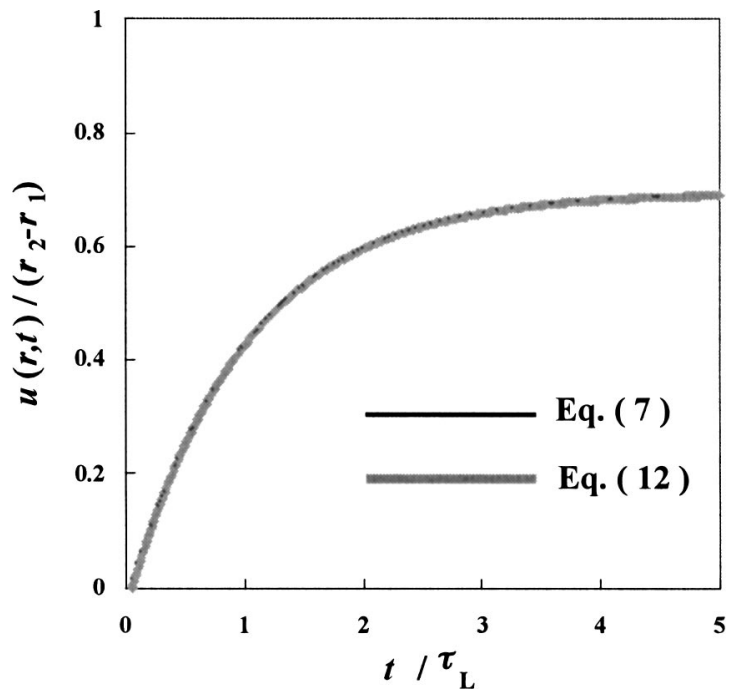

FIG. 4. The comparison of $u(r, t)$ given by Eqs. (7) and (12). The time is scaled by $\tau_{L}$. The former is calculated by summing the terms up to $n$ $=1000$. No appreciable difference is present between the two curves over the whole time region. The curves are calculated with $\alpha=4.5 \times 10^{4} \mathrm{G}$ and the same parameter values as Fig. 3 .

$\alpha$ increases, each of the initial shrinking rate and the stationary shrinkage increases as expected from Eqs. (10) and (11), respectively.

Although the exact solution of $u(r, t)$ is a multiexponential function shown by Eq. (7), the solution is well approximated by a single exponential function just with the leading term for $n=1$ : The contributions of the high-order terms $(n \geqslant 2)$ proportional to $1 /(2 n-1)^{3}$ are negligibly small relative to that for $n=1$. Thus the expression of $u\left(r_{1}, t\right)$ is reasonably simplified as

$$
\begin{aligned}
u\left(r_{1}, t\right) \approx & \frac{16 a^{2}}{\pi^{4}} \frac{\phi \Delta \rho \omega^{2}}{L}\left(2 a-\pi r_{2}\right) \exp \left(-\frac{t}{\tau_{L}}\right) \\
& +u\left(r_{1}, \infty\right) .
\end{aligned}
$$

It is seen in Fig. 4 that there exists no noticeable difference in the whole time dependence of $u\left(r_{1}, t\right)$ given by Eqs. (7) and (12).

\section{EXPERIMENT}

Poly(acrylamide) (PAAm) gels were prepared by radical copolymerization of acrylamide monomer and methylenebisacrylamide (crosslinker) employing ammoniumpersulfate as an initiator. The mixtures were dissolved in water. The monomer concentration was $3.00 \mathrm{wt} . \%$, and the molar ratio [monomer]/[crosslinker] was 100 . After the reactant solution was poured into an optical cell for the ultracentrifugal measurement, the cell was maintained at $5{ }^{\circ} \mathrm{C}$ for $24 \mathrm{~h}$ for crosslinking reactions.

Measurements were made using an analytical ultracentrifuge (Beckman, Model E) with a light of $\mathrm{Hg}$ e-line (546 $\mathrm{nm})$ at $25^{\circ} \mathrm{C}$. A single-sector $12-\mathrm{mm}$ cell with quartz glass window was employed. The centerpiece of the cell is made of poly(trifluorochloroethylene) to minimize the friction between the cell wall and the gel. The initial dimension of the 
gel in the centrifugal direction is $\approx 9 \mathrm{~mm}$, and the thickness and width, both of them are unchanged under centrifugal fields, are $\approx 12$ and $4 \mathrm{~mm}$, respectively. The measurements were carried out at the rotor speed of $1.80 \times 10^{4}$ or 6.00 $\times 10^{4} \mathrm{rpm}$. The Schlieren diagrams photographed were read on a contour projector (Nippon Kogaku, L-16) to the accuracy of $\pm 0.001 \mathrm{~mm}$.

As the value of $\rho_{N}$ for PAAm networks is unknown, the density of amorphous linear PAAm $\left(\rho=1.445 \times 10^{3} \mathrm{~kg} / \mathrm{m}^{3}\right)$ was employed as $\rho_{N}$ needed for the evaluation of $\Delta \rho$. The density of the linear PAAm with $M_{w}=1.0 \times 10^{4} \mathrm{~g} / \mathrm{mol}$ was evaluated from the densities of the aqueous solutions with various PAAm concentrations assuming the additivity for volume.

\section{RESULTS AND DISCUSSION}

Figure 5 displays the Schlieren patterns during the shrinking of the PAAm gel at the centrifugal acceleration of $\alpha=2.26 \times 10^{4} \mathrm{G}$. In these patterns, each position of airsolvent and solvent-gel interfaces is evidently recognizable as the point where the concentration gradient diverges. At $t$ $=6.00 \times 10^{2} \mathrm{~s}$, the air-solvent and solvent-gel interfaces are not distinguishable because the rotation time is too short to yield a finite shrinking. At $t=9.38 \times 10^{4} \mathrm{~s}(\approx 1$ day $)$, an appreciable shift of the solvent-gel interface toward the cell bottom (i.e., shrinking) is visible, while the air-solvent interface is immobile due to the incompressibility of the solvent. After sufficiently long time to achieve the stationary state $\left(t=6.95 \times 10^{5} \mathrm{~s}\right)$, the length of the gel decreases to less than a half of the initial length. It should be noticed in the last column in Fig. 5 that the position of the solvent-gel interface shifted by the centrifugal force slowly returns to the original position after stopping the rotation. This indicates that the deformation induced by centrifugal forces is recoverable; that no irreversible structural change in the gel is caused by the centrifugal forces. A finite concentration gradient observed between the air-solvent interface and the cell bottom primarily stems from the unreacted monomers which is originally present inside the gel. The time course of the change in the concentration gradient may reflect the sedimentation process of the unreacted monomers inside the gel, but it is beyond the scope of the present study to enter into the details.

Part (a) of Fig. 6 illustrates the position of the moving surface of the gels (i.e., the gel-solvent interface) as a function of time at $\alpha=r_{i} \omega^{2} / g=2.26 \times 10^{4}$ or $2.57 \times 10^{5} \mathrm{G}$. The value of $r$ at $t=0\left(r_{i}\right)$ corresponds to the initial distance of the gel surface from the rotation center before applying the centrifugal force. In the case of $\alpha=2.26 \times 10^{4} \mathrm{G}$, the shrinking proceeds linearly with time in the short time region, while the steady state of shrinking is achieved in the long time region (after $\approx 6$ days). The extrapolation from the data in the short time region to $t=0$ slightly deviates from the data at $t=0$, resulting from the fact that a finite time $(\approx 7$ $\min$ ) is required to reach the constant rotation speed in the experiments. Thus we employ the intercept at $t=0$ in the linear extrapolation as $r_{i}$ for further analysis. At the higher centrifugal acceleration of $\alpha=2.57 \times 10^{5} \mathrm{G}$, the shrinkage also increases linearly with time, and the shrinking rate is

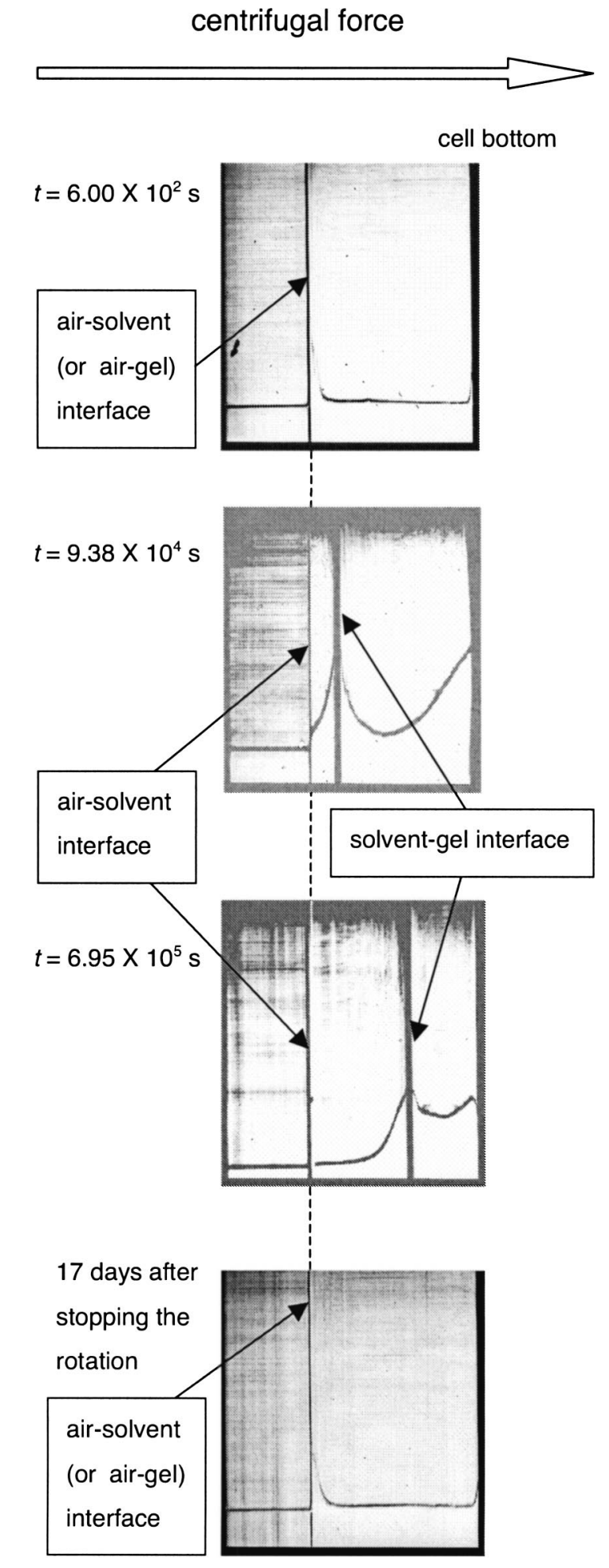

FIG. 5. The Schlieren patterns in the shrinking process of a PAAm gel at the centrifugal acceleration $\alpha=2.26 \times 10^{4} \mathrm{G}$ as a function of time. The last column shows an example of the patterns sufficiently long after stopping the rotation.

about 1 order of magnitude larger than that at $\alpha=2.26$ $\times 10^{4} \mathrm{G}$. Meanwhile, the true value of stationary shrinking is not attainable at $\alpha=2.58 \times 10^{5} \mathrm{G}$, because the high centrifugal force yields such a large shrinking that the shift of the gel surface stops due to the presence of the bottom wall (located at $r=71.76 \mathrm{~mm}$ ). 


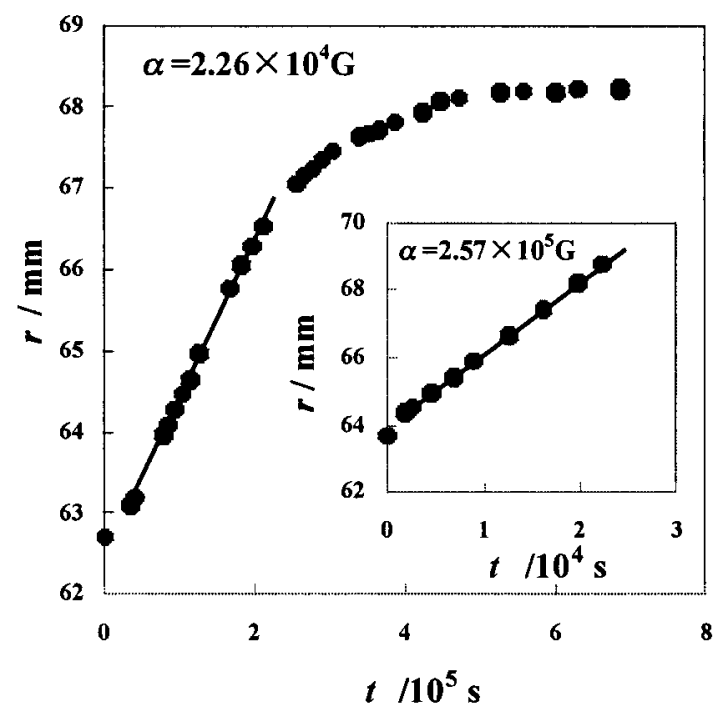

(a)

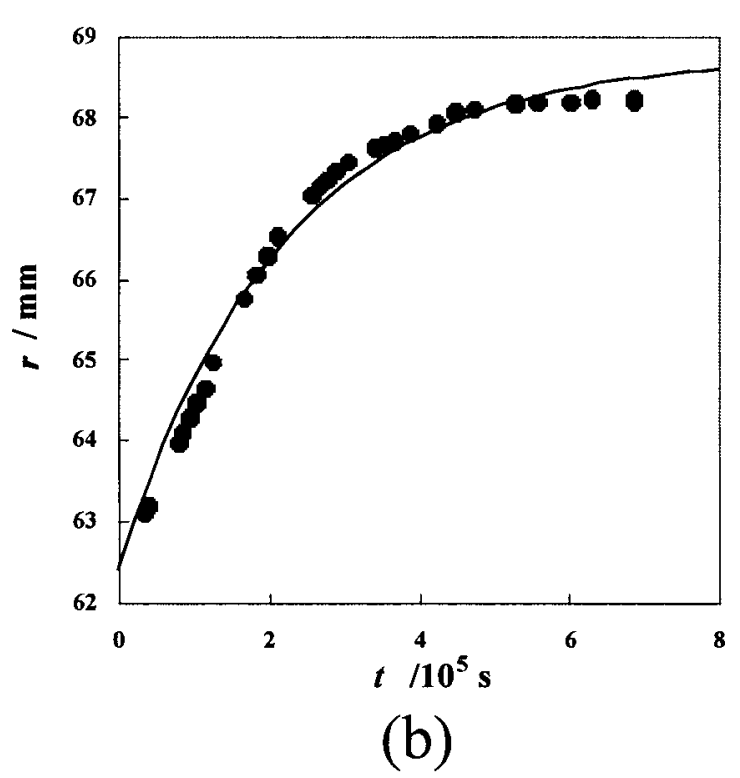

FIG. 6. (a) Position of the solvent-gel interface (the moving surface) of a PAAm gel as a function of time at the centrifugal acceleration of $\alpha=2.26$ $\times 10^{4} \mathrm{G}$. The inset shows the same plots for the data at $\alpha=2.57 \times 10^{5} \mathrm{G}$. The straight lines represent the linear regression in the short time region. The deviations of the data at $t=0$ from the linear extrapolations result from the fact that a finite time $(\approx 7 \mathrm{~min})$ is required to achieve each constant rotation speed. (b) The comparison of the experimental data at $\alpha=2.26$ $\times 10^{4} \mathrm{G}$ with the best-fitted curve of Eq. (12). The fitted values of $\tau_{L}$ and $L$ are $2.16 \times 10^{5} \mathrm{~s}$ and $1.50 \times 10^{4} \mathrm{~Pa}$, respectively.

According to Eqs. (10) and (11), the modulus $L$ and the friction coefficient $f$ can be evaluated from the stationary shrinkage and the initial shrinking rate, respectively. For the calculations, $r_{1}=6.240 \times 10^{-2} \mathrm{~m}$ and $r_{2}=7.174 \times 10^{-2} \mathrm{~m}$ for $\alpha=2.26 \times 10^{4} \mathrm{G} ; \quad r_{1}=6.395 \times 10^{-2} \mathrm{~m}, \quad$ and $\quad r_{2}=7.176$ $\times 10^{-2} \mathrm{~m}$ for $\alpha=2.57 \times 10^{5} \mathrm{G}$ were used together with $\phi$ $=2.09 \times 10^{-2}$ and $\Delta \rho=\rho_{N}-\rho_{\text {water }}=4.48 \times 10^{2} \mathrm{~kg} / \mathrm{m}^{3}$. From Eq. (11) and the linear regression for the data in the short time region at $\alpha=2.26 \times 10^{4}$ and $2.58 \times 10^{5} \mathrm{G}, f$ is estimated to be $1.04 \times 10^{14}$ and $1.10 \times 10^{14} \mathrm{Ns} / \mathrm{m}^{4}$, respectively. The data at different $\alpha$ yield almost the same value of $f$, indicat- ing that the initial shrinking rate is proportional to $\alpha$ as expected from the theory. These $f$ values are also close to $f$ $=1.5 \times 10^{14} \mathrm{Ns} / \mathrm{m}^{4}$ obtained by a frictional measurement ${ }^{8}$ of the PAAm gel with the same concentrations of monomer and crosslinker as the PAAm gel in the present study. This agreement indicates the validity of the theory in the present study, and it also assures that the effect of the friction between the cell wall and the gel on shrinking is negligibly small. From Eq. (11) with $r_{\infty}=6.820 \times 10^{-2} \mathrm{~m}$ at $\alpha=2.26 \times 10^{4} \mathrm{G}, L$ is evaluated to be $1.64 \times 10^{4} \mathrm{~Pa}$. These values of $f$ and $L$ yield $D=1.57 \times 10^{-10} \mathrm{~m}^{2} / \mathrm{s}$.

Part (b) of Fig. 7 displays the result of the curve fit based on Eq. (12) to the whole time dependence of the data at $\alpha$ $=2.26 \times 10^{4} \mathrm{G}$. In the fitting, $\tau_{L}$ and $L$ were used as adjustable parameters while other parameter values were the same as aforementioned values. The theoretical curve satisfactorily fits the data, although each of the initial shrinking rate and stationary shrinkage in the fitted curve is slightly larger than that in the data. The fitted values of $\tau_{L}$ and $L$ are 2.16 $\times 10^{5} \mathrm{~s}$ and $1.50 \times 10^{4} \mathrm{~Pa}$, respectively. These yield $f=9.17$ $\times 10^{13} \mathrm{Ns} / \mathrm{m}^{4}$ and $D=1.64 \times 10^{-10} \mathrm{~m}^{2} / \mathrm{s}$. Each value of $L, f$, and $D$ obtained in the curve fitting well accords with that evaluated from the stationary shrinkage and initial shrinking rate as mentioned above, respectively.

Thus the experimental data on the shrinking of the PAAm gel under ultracentrifugal fields are well explained by the theory proposed here. Further, the present work has demonstrated that the ultracentrifugal measurements of gels enable us to evaluate simultaneously $f$ and $L$ of polymer networks.

\section{CONCLUSIONS}

Ultracentrifugal fields induces the shrinking of gels. In the short time region, the shrinking proceeds at a constant rate, while the shrinking reaches the stationary state in the long time limit. A theory is proposed to describe the diffusion process of networks in the presence of centrifugal fields. The theory demonstrates the following characteristics of the shrinking behavior: The initial shrinking rate is dominated by the ratio of the frictional force and the centrifugal force. The stationary shrinkage is determined by the balance between the swelling force and the centrifugal force. The whole shrinking process substantially obeys a single exponential relaxation whose characteristic time is proportional to the ratio of the square of the stationary displacement and the diffusion constant of networks. The whole shrinking process of a polyacrylamide gel under ultracentrifugal fields observed is satisfactorily described by the theory. The analysis based on the theory enables us to evaluate the friction constant as well as the longitudinal elastic modulus of the network.

${ }^{1}$ T. Tanaka, L. O. Hocker, and G. B. Benedek, J. Chem. Phys. 59, 5151 (1973).

${ }^{2}$ T. Tanaka and D. J. Fillmore, J. Chem. Phys. 70, 1214 (1979).

${ }^{3}$ A. Peters and S. J. Candau, Macromolecules 21, 2278 (1988).

${ }^{4}$ Y. Li and T. Tanaka, J. Chem. Phys. 92, 1365 (1990).

${ }^{5}$ A. Onuki, in Responsive Gels: Volume Transition I, edited by K. Dusek, Adv. Polym. Sci. (Springer-Verlag, Berlin and Heidelberg, 1993), Vol. 109, p. 63. 
${ }^{6}$ C. Wang, Y. Li, and Z. Hu, Macromolecules 30, 4727 (1997).

${ }^{7}$ T. Yamaue and M. Doi, Phys. Rev. E 69, 041402 (2004).

${ }^{8}$ M. Tokita and T. Tanaka, J. Chem. Phys. 95, 4613 (1991).

${ }^{9}$ T. Takigawa, K. Uchida, K. Takahashi, and T. Masuda, J. Chem. Phys. 111, 2295 (1999).

${ }^{10}$ T. Takigawa, K. Urayama, Y. Morino, and T. Masuda, Polym. J. (Tokyo, Jpn.) 25, 929 (1993).

${ }^{11}$ T. Takigawa, K. Urayama, and T. Masuda, Polym. Gels Networks 2, 59 (1994).
${ }^{12}$ K. Urayama, T. Takigawa, and T. Masuda, Rheol. Acta 33, 89 (1994).

${ }^{13}$ A. Suzuki and H. Hara, J. Chem. Phys. 114, 5012 (2001).

${ }^{14}$ S. Sasaki, J. Chem. Phys. 120, 5789 (2004).

${ }^{15}$ S. Hirotsu, Macromolecules 37, 3415 (2004).

${ }^{16}$ T. Takigawa, S. Nosaka, Y. Takakura, and K. Urayama, Polym. J. (Tokyo, Jpn.) 35, 819 (2003).

${ }^{17}$ L. D. Landau and E. M. Lifshitz, Theory of Elasticity (Pergamon, Oxford, 1986). 